\title{
A Silicon-Based Tactile Sensor for Finger-Mounted Applications
}

\author{
David J. Beebe,* Member, IEEE, Denice D. Denton, Member, IEEE, Robert G. Radwin, Senior Member, IEEE, \\ and John G. Webster, Life Fellow, IEEE
}

\begin{abstract}
This paper presents a silicon-based force sensor packaged in a flexible package and describes the sensors performance on human subjects. The sensing element consists of a circular silicon diaphragm $(200-\mu \mathrm{m}$ thick with a $2-\mathrm{mm}$ radius) over a 10- $\mu \mathrm{m}$ sealed cavity with a solid Torlon dome providing force-to-pressure transduction to the diaphragm. Two dome heights $(0.5$ and $1.5 \mathrm{~mm})$ were compared. The sensor with the taller dome showed improved sensitivity. Dynamic calibration and tracking experiments are performed with the sensor mounted on the dominant thumb of five human subjects. Both force and loading direction are statistically significant $(P<\mathbf{0 . 0 5})$. Subject variability accounted for $8.7 \%$ of the variance, while loading direction accounted for $1.9 \%$ of the variance. Average errors for the tracking experiment range from -2.8 to $1.0 \mathrm{~N}$ and are subject dependent. Three out of four subjects showed increasing negative error with increasing load.
\end{abstract}

Index Terms - Pinch force, sensor, silicon, tactile.

\section{INTRODUCTION}

\section{A. Need/Requirements}

$\mathbf{T}$ HE need for tactile sensors is well documented. Several books and papers describe the requirements and applications for tactile sensors [1]-[4]. A wide variety of applications exist for a sensor which can accurately measure finger and hand forces. Practical finger and hand force sensors are needed for biomechanics research, clinical evaluations of hand function, and for hand rehabilitation devices. Measurement and prediction of palmar force distribution during grip functions are important for developing functional biomechanical models and for designing tools, work equipment and manual activities. Force conveys information concerning stresses on the muscles and tendons used for grip. A major limitation in accurately predicting internal hand forces using biomechanical models is determining the actual external finger forces applied during different grip configurations and hand functions[5]. Hand force sensors are also needed for evaluating the effects of surgical

Manuscript received June 3, 1996; revised July 2, 1997. This work was supported by the Naitonal Institutes of Health under Grant 5T32GMO8349. Asterisk indicates corresponding author.

*D. J. Beebe is with the Department of Electrical and Computer Engineering, The Beckman Institute, University of Illinois at Urbana-Champaign, 405 North Matthews Avenue, Urbana, IL 61801 USA (e-mail: dbeebe@uiuc.edu)

D. D. Denton is with the Department of Electrical Engineering, University of Washington, Seattle, WA 98195 USA.

R. G. Radwin is with the Department of Industrial Engineering, University of Wisconsin-Madison, Madison, WI 53706 USA.

J. G. Webster is with the Department of Electrical and Computer Engineering, University of Wisconsin-Madison, Madison 53706 WI USA.

Publisher Item Identifier S 0018-9294(98)00905-7. techniques such as joint replacement and tendon transfers [6]. In addition, tactile sensors could be used in rehabilitation and for functional testing. For example, accurate measurement of hand forces would aid in documenting the progress of hand surgery patients. Existing functional measuring techniques are time consuming and often force the hand into unnatural grasps. The development of a small, reliable force sensor which could be distributed about the palm would greatly improve a surgeon's ability to plan and accurately evaluate a patient's progress in reconstructive surgery. Accurate force sensors are urgently needed for use with functional neuromuscular stimulation (FNS) [7]. Tactile sensors are necessary to provide force information feedback in FNS systems. Sensory substitution rehabilitation devices that compensate for loss of sensation in the hand are another area in need of tactile sensors [8]. Other areas which would benefit from the development of a tactile sensor include robotics, teleoperator systems, prosthetics, and the measurement of hand forces of workers.

\section{B. Tactile Sensors}

The development of a finger-mounted tactile sensor which meets the requirements necessary for typical applications has been elusive. Usable force sensors have been developed [4], [9], but a true tactile sensor has not. Many types of commercially available sensors have been investigated to some degree for applications in tactile sensing [3], [10]. Although technology has rapidly progressed in the area of robotic end effector sensors, few researchers have attempted to specifically address the problems associated with tactile sensing in a clinical setting. Recent technological advances have provided small, thin sensors having promise for use in directly measuring individual finger forces during normal grasping activities. However, conventional force measurement sensors are inadequate for measuring hand forces produced during manual work activities and activities of daily living. Either they are too large and bulky for attaching directly to the hands or fingers without significantly degrading manual dexterity, or they are too fragile to withstand the high forces exerted by the hand.

Reston and Kolesar [11] have constructed a $5 \times 5$ robotic tactile sensor based on piezoelectric polyvinylidene fluoride (PVDF) film coupled to an integrated circuit. Although the array provided a linear output, its limited range and inability to measure static forces makes it a poor choice for a fingermounted tactile sensor. Germagnoli et al. [12] developed a fingertip $8 \times 6$ array sensor sensitive to all six independent 
stress tensors (based on previous work by De Rossi et al. [13]) by careful orientation of PVDF sensing elements. The sensor sensitivity is low and requires amplification close to the sensor to achieve an adequate signal-to-noise ratio. Fellows and Freivalds [14] attached conductive polymer sensors to a garden-tool handle. Although these sensors are durable, they are pressure sensitive devices rather than true force sensors [15]. A conductive polymer force sensor for attaching to the palmar surface of the hand has been developed and rigorously tested [9]. Kaczmarek, et al. [16] has developed an electrotactile stimulation system which utilizes a glove with conductive polymer pressure sensors. However, these sensors are inaccurate and exhibit significant hysteresis. Although somewhat limited in range and resolution, these sensors are highly durable and practical for measuring individual finger forces exerted during submaximal pinch [17]. The Lord Industrial Automation (Covy, NC) sensor is a commercially available optical force sensor [18]. However, the sensors are too thick $(28.4 \mathrm{~mm})$ to be useful in finger-mounted applications. Neuman et al. [19] have developed a multielement capacitive sensing array. In actual use, crosstalk due to mechanical coupling between the capacitors resulted in significant errors. Temperature sensitivity $(<1.35 \mathrm{~N} / \mathrm{C})$ and drift $(1.28 \mathrm{~N} / \mathrm{h})$ were also significant. Commercially available force sensors based on strain gages are widely available. They are more commonly called load cells. However, few are small enough to be used for tactile sensing. Those that are relatively small are expensive.

\section{Silicon Tactile Sensors}

Many silicon force sensors have been reported in recent years and silicon pressure sensors are widely available commercially, but are not useful as finger-mounted tactile because they: 1) are packaged in a bulky, hard thermoplastic case, 2) measure pressure, not force, and 3) lack over-load protection. Suzuki et al. [20] developed a silicon tactile imager based on an array of capacitive cells. Samaun et al. [21] developed an early piezoresistive-pressure sensor which could be catheter mounted. Lee and Wise [22] developed a silicon capacitivepressure sensor. Tanigawa et al. [23] developed a silicon pressure sensor with on-chip signal processing. Kane and Kovacs [24] report a tactile sensor capable of high-resolution imaging. The sensor uses a CMOS compatible process and can resolve both normal and shear stress. However, all of these sensors have a thin diaphragm and most target the problem of tactile imaging and are, thus, unsuitable for measuring finger and hand forces in clinical applications. Chu et al. [25] developed a silicon-based three-axial tactile sensor that holds considerable promise, but it is packaged using traditional integrated circuit (IC) mounting and wire bonding techniques. Sorab et al. [10] developed a system for measuring fingertip-applied forces during delivery of newborns, using piezoresistive silicon sensors that were placed on the clinician's fingertips. These sensors lacked durability and were not suitable for measuring forces during manual work and activities of daily living. Ko et al. [26] and Neuman et al. [27] developed a piezoresistive-pressure sensor and tested it on human subjects. Crago et al. [4] discuss the limitations of the sensor due to the size and stiffness of the sensor with respect to the finger. They conclude that to determine forces against a finger more accurately, a sensor must cover more of the finger and it must conform to its shape.

The sensor presented here addresses these shortcomings by focusing on flexible packaging, thick diaphragm, and over-force protection. The polyimide-based packaging scheme provides for a durable, flexible package which can be finger mounted. Since the details of the package durability testing and device fabrication are covered elsewhere [28], [29], this paper will provide only brief description of the overall fabrication process and focus on the characteristics of the finished sensor when mounted on human subjects.

\section{DEVICE DESIGN AND FABRICATION}

Barth et al. [30] developed a polyimide-based package for silicon temperature sensors. The package worked well for temperature profile measurements during hyperthermia treatment of cancer, but was not durable enough to withstand the rigors of tactile-sensing applications. These shortcomings have been overcome via the development of a flexible polyimide-based package, the addition of a solid dome which acts as a forceto-pressure transducer over the diaphragm and the use of a very shallow cavity under the diaphragm to provide over-force protection. The modifications to Barth's package described here create a new package durable enough for use as a fingermounted sensor. The tactile sensor is similar to a Band-Aid, where the gauze pad is the force sensor and the adhesive strip is a flexible runner for the electrical leads. An illustration of the sensor system is shown in Fig. 1(a)-(c). The finger-mounted sensor is shown in Fig. 3(a). Note, the flexible leads allow the signal and power wires to be attached at the back of the finger away from the applied force. The connection to the silicon sensing element is an integral part of the packaging process, thus, the connection is flat and well protected (as compared to traditional IC wire-bonding techniques).

The sensor design is based on a silicon diaphragm structure instrumented with ion-implanted piezoresistors in a Wheatstone bridge configuration. The applied force is distributed across the diaphragm via the solid dome. The distributed force deforms the diaphragm giving rise to an output voltage proportional to the applied force for small deflections. At high forces, the diaphragm deflection is restricted by the cavity bottom limiting the maximum stress and extending the useful range of the sensor.

The fabrication process utilizes a combination of standard IC fabrication techniques and bulk micromachining techniques and is described in detail elsewhere [29]. A brief description is given here for completeness. Shallow (10- $\mu \mathrm{m}$ deep) cavities are into a double-side polished wafer etched using potassium hydroxide and silicon fusion bonding is used to bond this wafer to a single-side polished wafer forming an array of sealed cavities. Piezoresistors are ion implanted at the edges of the diaphragm (two tangential and two radial resistors are used). A polyimide/metal/polyimide sandwiched lead structure is then used to connect the resistors in a Wheatstone bridge 


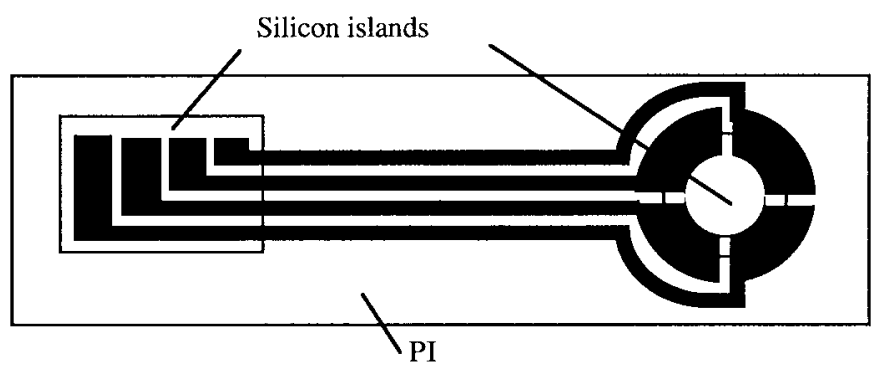

(a)

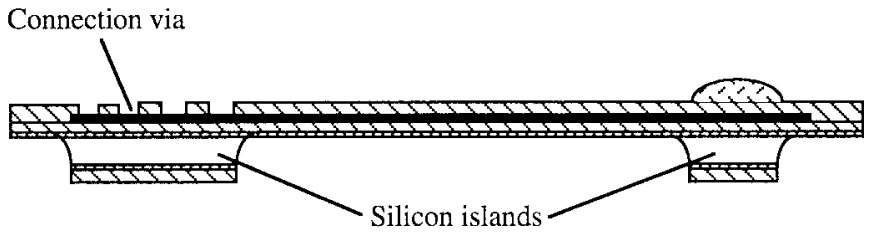

Al leads

피 Cr adhesion layer

Implanted piezoresistors

[ज] Polymer dome

Not to scale

(b)

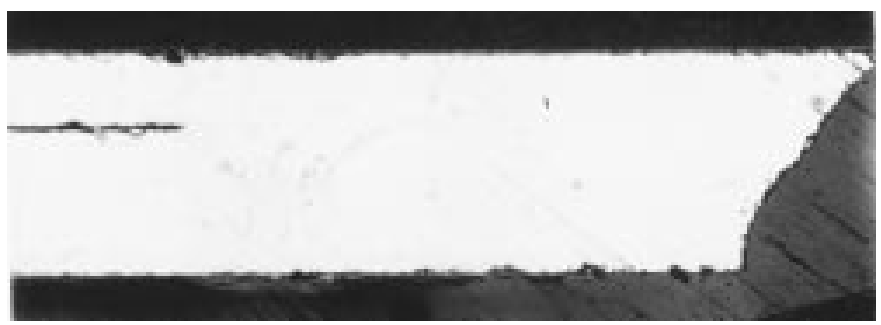

(c)

Fig. 1. (a) Top view of package sensor illustrates arc lead pattern designed to increase durability. (b) Side view illustrates the sandwiched lead structure. (c) A cross-sectional view of the one side of a sensing island showing the $200-\mu \mathrm{m}$-thick diaphragm and isotropic etch profile.

configuration. An isotropic bulk etchant is used to remove the majority of the silicon leaving two silicon islands per sensor. A circular island contains the sensing element. A rectangular island at the opposite end of the finished sensor package provides a rigid substrate to prevent lead delamination and to facilitate connector attachment. A Torlon dome is fabricated using traditional milling techniques and is attached over the diaphragm to provide force-to-pressure transduction between the applied load and the silicon diaphragm [29]. An epoxy bead is manually applied at all interfaces between the rigid silicon islands and the flexible polyimide leads. A thin layer of urethane is manually spread over the entire package to further enhance durability. The finished sensors are then removed using a razor blade. An illustration of a finished sensor is shown in Fig. 1(a) and (b) and a photograph showing a crosssectional view of part of the diaphragm, cavity and isotropic etch profile is shown in Fig. 1(c).

Previous work demonstrated that increases in lead width provided increased durability [28]. As an extension of this result, a new lead style was designed to maximize the area of lead coverage at the silicon/polyimide interface. The lead style is shown in Fig. 1.

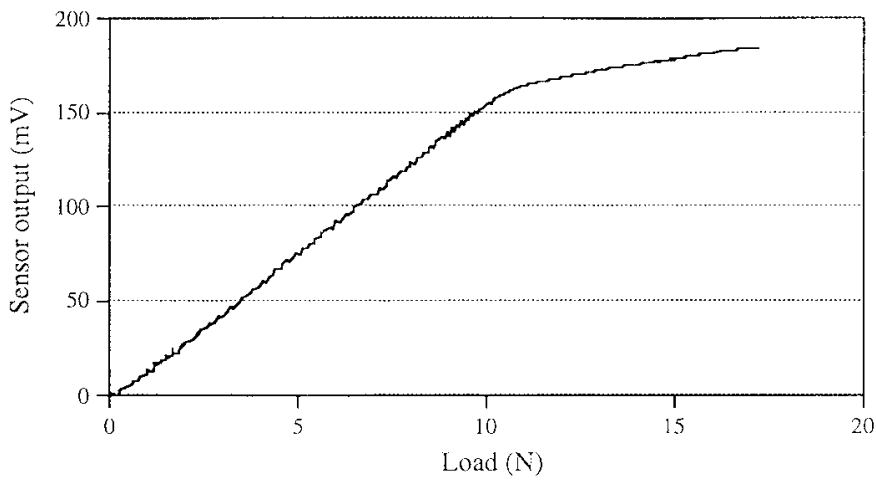

Fig. 2. With the sensor mounted on a rigid substrate (i.e., bench testing), the performance is excellent with negligible hysteresis and good linearity prior to the diaphragm making contact with the bottom of the cavity.

\section{EXPERIMENTAL METHODS}

\section{A. Bench Testing}

The sensor was tested in two configurations: 1) mounted on a rigid substrate (i.e., bench testing), and 2) finger-mounted. The performance of the sensor in response to a variety of loads when mounted on a rigid substrate is described in detail elsewhere [29]. A typical dynamic calibration curve for the bench-mounted sensor is shown in Fig. 2. Note the hysteresis is negligible and linearity $(R=0.996)$ is good up to the point where the diaphragm deflection is limited by the cavity bottom.

\section{B. Method of Sensor Attachment}

While the performance of the sensor when mounted on a rigid substrate is of interest, many applications require that the sensor be mounted on less rigid substrates. For FNS uses, finger-mounting is required. To examine the usefulness of this sensor in these types of applications, finger-mounted tests were performed. By pinching the fingers together and observing the skin's deformation and subsequent return to its unloaded state, it is easy to observe that the human finger is less than ideal as a substrate for sensor mounting. It has hysteresis and is nonlinear [31]-[33]. For all finger-mounted tests the sensor was mounted on the pad over the distal phalanx on the thumb of the dominant hand as shown in Fig. 3(a). The sensor was secured with Duraspore tape. To further secure the sensor and to hold the wires in place a thin, tight-fitting nylon glove (MEDTECH International, The DRYGARD Glove Liner, Altamonte Springs, FL) was used as shown in Fig. 3(b).

\section{Force Transmission (Dome Size)}

In order to measure force using a pressure-sensitive device, an additional structure is required over the diaphragm so that any applied force is distributed across the diaphragm. A solid dome structure was chosen for this study. Two dome shapes where investigated as shown in Fig. 4. The choice of Torlon, a polyamide-imide, was based on ease-of-use, stiffness, and machinability. The Torlon domes are attached to the diaphragm using cyanoacrylate ester (Tru-Bond, True Value, Chicago, IL). 


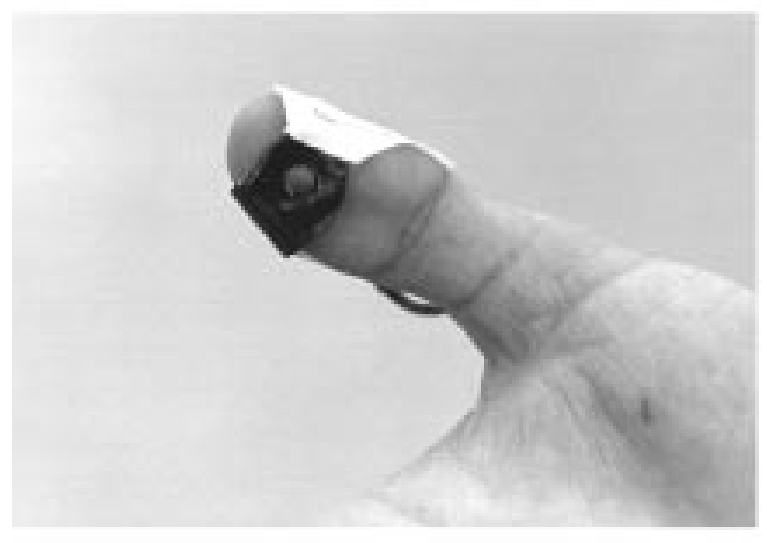

(a)

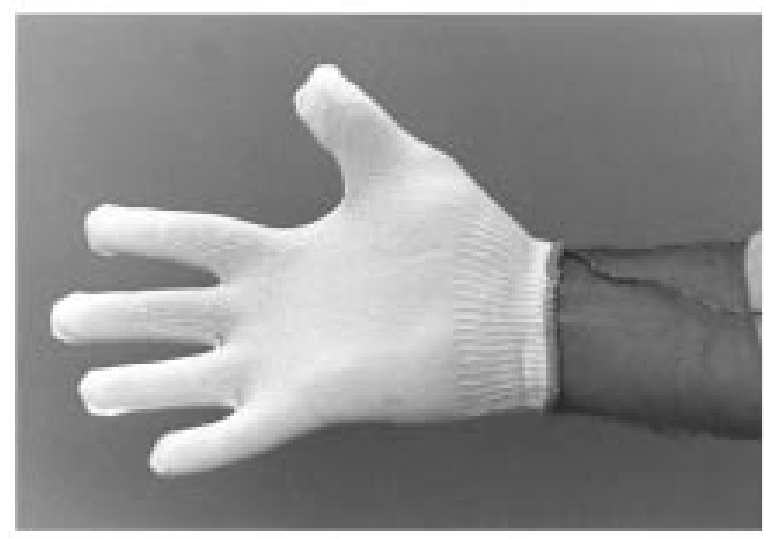

(b)

Fig. 3. (a) The thumb-mounted sensor is about the size of a band-aid. (b) A tight-fitting nylon glove was worn over the sensor to secure the sensor in the proper position on the pad of the thumb.

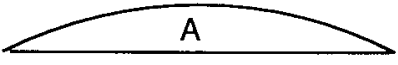

$\mathrm{h} \quad=0.5 \mathrm{~mm}$

r $\quad=4.25 \mathrm{~mm}$

$\mathrm{d}=4 \mathrm{~mm}$.

$\operatorname{arc}=56.14^{\circ}$

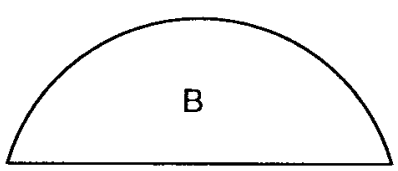

$\mathrm{h} \quad=1.5 \mathrm{~mm}$

$\mathrm{r}=2.083 \mathrm{~mm}$

$\mathrm{d}=4 \mathrm{~mm}$

$\operatorname{arc}=147.5^{\circ}$
Fig. 4. Two dome structures were designed. Both cover the entire surface of the diaphragm to provide enhanced protection from point loads.

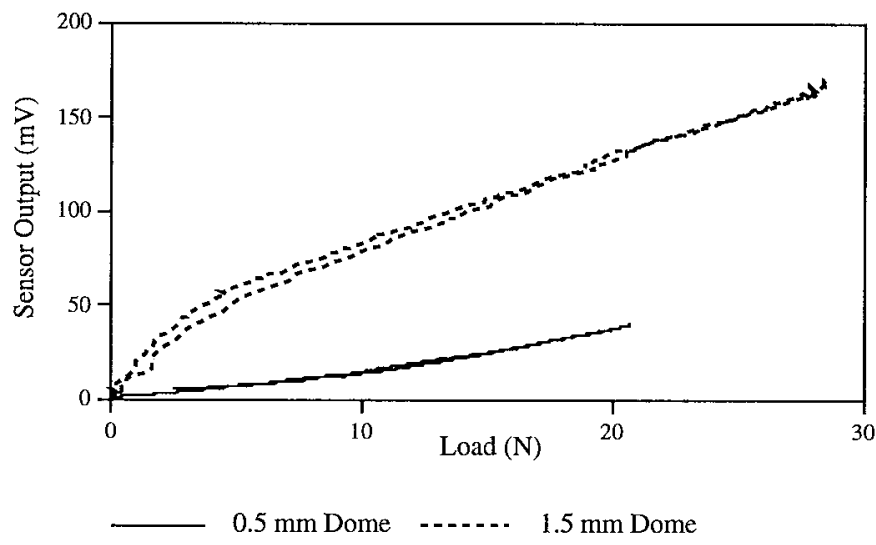

Fig. 5. Dome height has a significant effect on the sensor response.

As shown in Fig. 5, the dome size has an effect on the performance of the sensor in finger-mounted applications. The output is much greater when using a taller dome. The taller $(1.5 \mathrm{~mm})$ dome was used for all subsequent finger-mounted tests.

\section{Dynamic Calibration}

Dynamic calibration was performed on five subjects (one female, four male) varying in age from 24-62 y. A dual beam strain gage dynamometer was used as a reference and held between the thumb and forefinger for both tests. The dynamometer output was independent of load location along the beam [34]. The subject was instructed to slowly increase the pinch force to a maximum voluntary level and then to slowly release the pinch. The maximum level was defined as the force level at which the subject was willing to hold for at least $10 \mathrm{~s}$. The subject was free to use as many opposing fingers as desired. In addition, the subject released their pinch and repositioned their fingers between each cycle. This led to increased variability, but it was representative of the way people grasp objects in daily living (i.e., grip sometimes varies even when grasping the same object). Five repetitions were performed on each subject. The first repetition was treated as practice and was not included in the analysis. Typical response curves are shown in Fig. 6(a) and (b) with the sensor output $(\mathrm{mV})$ on the $y$ axis and the applied force $(N)$ or time $(s)$ on the $x$ axis.

Calibration results were studied using analysis of variance to evaluate the differences between the means of four force levels $(4,8,12,16 N)$. The $F$-ratio $(F)$ is computed as the mean square (sum-of-squares divided by the degrees of freedom) divided by the mean square error (MSE). The percentile of the $F$ distribution $(p)$ is used to indicate the probability of rejecting the null hypothesis when it is true, or to determine the statistical significance of the effect [35]. Significance levels of less than 0.05 were considered statistically significant. The percent of the variance due to a particular factor can be determined by dividing the sum of squares for each factor of interest by the total sum of squares. While a zero-load offset voltage was present, the offset voltage was subtracted from all data to reflect zero output under no-load conditions for clarity and comparison purposes.

The main effects of force and loading direction (i.e., increasing or decreasing load) are statistically significant $(P<0.05)$, as is the loading direction $\times$ force interaction. The force effect accounted for $85.25 \%$ of the variance. Subject variability accounted for $8.7 \%$ of the variance, while loading direction accounted for $1.9 \%$ of the variance. The MSE accounts for less than $0.18 \%$ of the variance $(\sqrt{\mathrm{MSE}} \approx 2.8 \mathrm{mV})$. This error term includes variance due to systematic errors. Overall the finger-mounted results show a great deal of promise for this sensor. For the dynamic calibration experiment, the sensor responded largely to force ( $85 \%$ of the variance). Hysteresis 


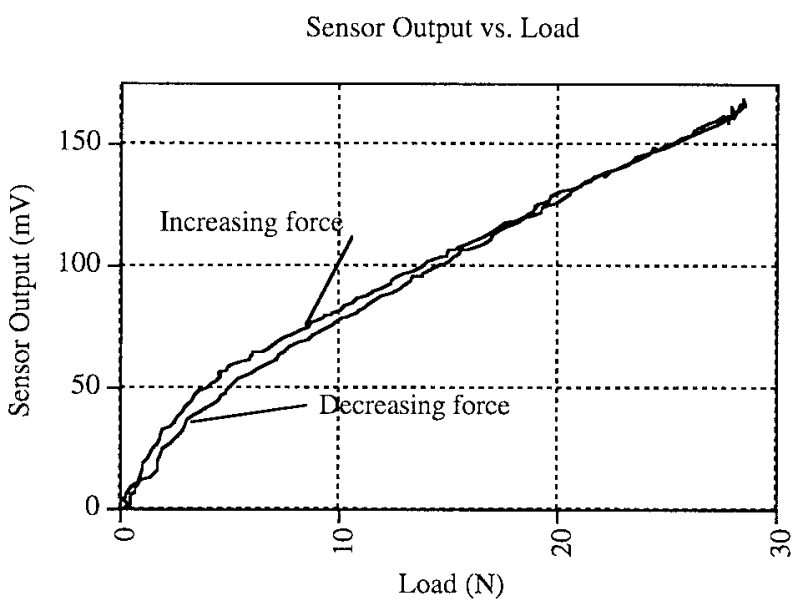

(a)

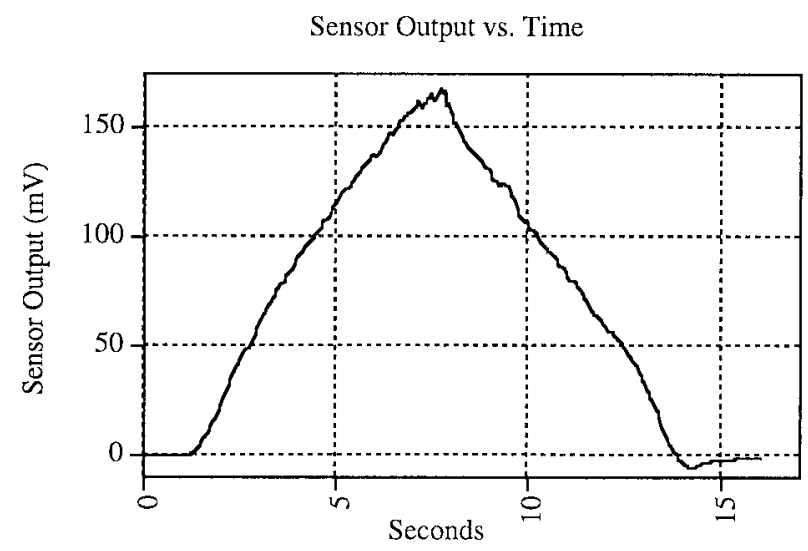

(b)

Fig. 6. (a) A typical finger-mounted response curve shows some nonlinear behavior at small forces, with a small amount of hysteresis. (b) For the dynamic calibration tests, the subject was asked to slowly increase and then slowly decrease their pinch force.

was small $(<6.7 \mathrm{mV})$ and repeatability on a given subject was excellent $(<0.2 \%$ of the variance). The analysis of variance (ANOVA) results are summarized in Tables I and II. Table III provides additional subject data and sensor means.

\section{E. Tracking Experiment}

To further evaluate the finger-mounted performance of the sensor, a tracking experiment was performed using four subjects. Each subject was asked to pinch the dynamometer to specific levels and hold that level of force for approximately 1-2 s. Visual force feedback information was provided to the subject on an oscilloscope. A run consisted of five consecutive pinch and hold patterns at five different force levels $(3,7,10$, 13, $16 N$ ). The order of the levels was randomized. Each run was repeated five times with the first repetition discarded. Fig. 7(a) and (b) shows typical sensor and dynamometer responses from two different subjects. Note that the accuracy of the sensor is dependent on the subject as illustrated in Fig. 8. Fig. 9 illustrates the error trends for each subject.

\section{F. Typical Task}

One application for the tactile sensors is the measurement of hand forces on factory workers to study repetitive-motion
TABLE I

ANALYSIS OF VARIANCE TABLE

\begin{tabular}{c|c|c|c}
\hline Source of variation & $\begin{array}{c}\text { Degrees of } \\
\text { freedom }\end{array}$ & $\mathrm{F}$ & $\mathrm{p}$ \\
\hline Force & 3 & 660 & 0 \\
\hline Direction & 1 & 10.4 & 0.03 \\
\hline Replicate & 3 & 1.4 & 0.3 \\
\hline Replicate $\times$ direction & 3 & 0.34 & 0.8 \\
\hline Replicate $\times$ force & 9 & 1.3 & 0.27 \\
\hline Direction $\times$ force & 3 & 34.2 & 0 \\
\hline Error & 36 & & \\
\hline
\end{tabular}

TABLE II

Percent Variance of Significant Sources of Variation

\begin{tabular}{c|c|c|c|c|c}
\hline Effect & Force & Subject & Direction & Replicate & Subject $x$ replicate \\
\hline \% variance & 85.3 & 8.7 & 1.9 & 0.15 & 1.9 \\
\hline
\end{tabular}

TABLE III

Summary of Mean Sensor Outputs and Subject Attributes

\begin{tabular}{c|c|c|c|c|c}
\hline & Subject 1 & Subject 2 & Subject 3 & Subject 4 & Subject 5 \\
\hline Mean output & 164.9 & 161.9 & 162 & 124 & 174.9 \\
\hline SD & 7.3 & 16.4 & 6.6 & 5.5 & 7.8 \\
\hline Finger circum. & $60 \mathrm{~mm}$ & $58 \mathrm{~mm}$ & $59 \mathrm{~mm}$ & $59 \mathrm{~mm}$ & $63 \mathrm{~mm}$ \\
\hline Finger width & $22 \mathrm{~mm}$ & $25 \mathrm{~mm}$ & $23 \mathrm{~mm}$ & $23 \mathrm{~mm}$ & $24 \mathrm{~mm}$ \\
\hline Age & 34 & 41 & 24 & 62 & 31 \\
\hline Sex & male & male & female & male & male \\
\hline
\end{tabular}

injuries. To illustrate the usefulness of this sensor in such applications a simple manual repetitive task was used. The subject was asked to press four film canister covers onto canisters one at a time. The canisters were fixed to a rigid substrate in a linear array with a spacing of $10 \mathrm{~cm}$. The subject was instructed to pick up a cover, place it on the canister, and push it until it was firmly seated. This was repeated until all four canisters were covered. The sensor was mounted on the thumb as described above. The subject was instructed to use his/her thumb to press the cover in place. Fig. 10 shows the force measured by the sensor during a typical run.

\section{DISCUSSION}

The sensor, when mounted on a rigid substrate (i.e., bench tested), has excellent performance characteristics. The performance of the finger-mounted sensor, however, is degraded. This is not surprising considering the nonlinear characteristics of the complex tissue/bone substrate present during the fingermounted tests. The discussion will focus on the performance of 


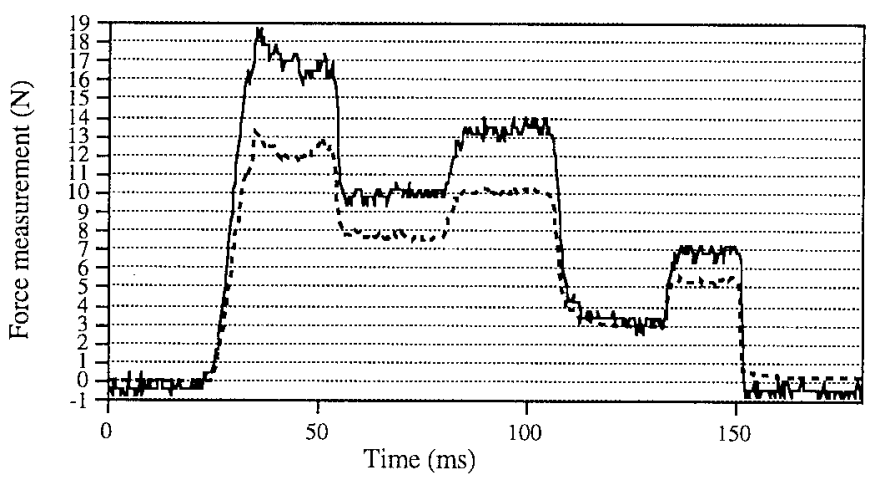

Load Cell $\ldots . . . .$. Sensor

(a)

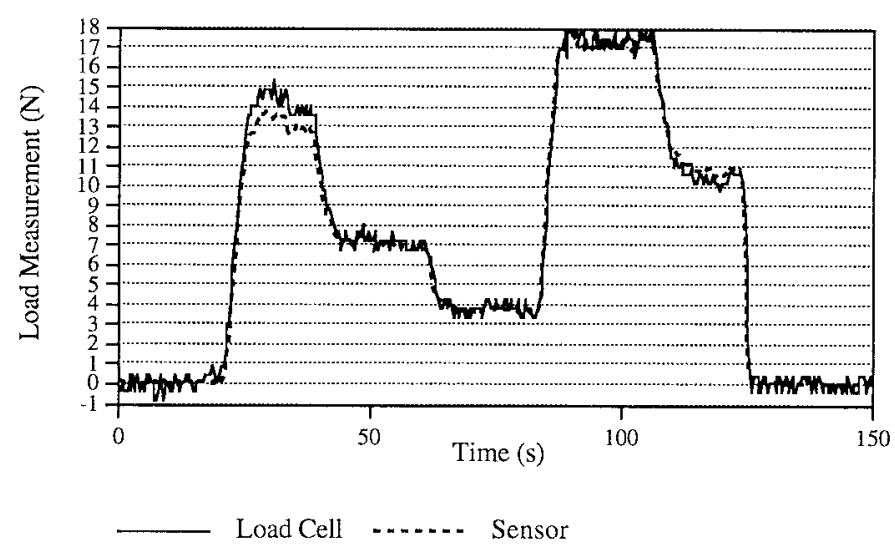

(b)

Fig. 7. The sensor output is very subject dependent: (a) subject 4 consistently registered low force levels and (b) subject 3 demonstrated more accurate output.

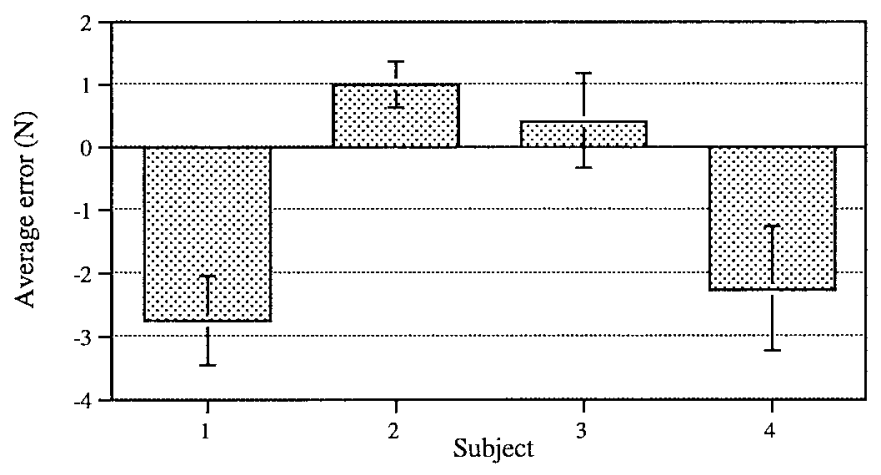

Fig. 8. The average error was subject dependent, but repeatability was good within a subject.

the sensor during the tracking experiments and how the sensor attributes (particularly, dome shape) affect performance.

\section{A. Dome Size}

Preliminary experiments with two different Torlon dome shapes showed markedly different effects on sensor calibration curves (see Fig. 5). The shape and general sensitivity of the sensor was different for each dome. The differences can be explained in terms of the mechanical interaction between the sensor and the finger and the relative sizes of the sensor and the finger. The rigid part of the sensor measures $8 \mathrm{~mm}$ in

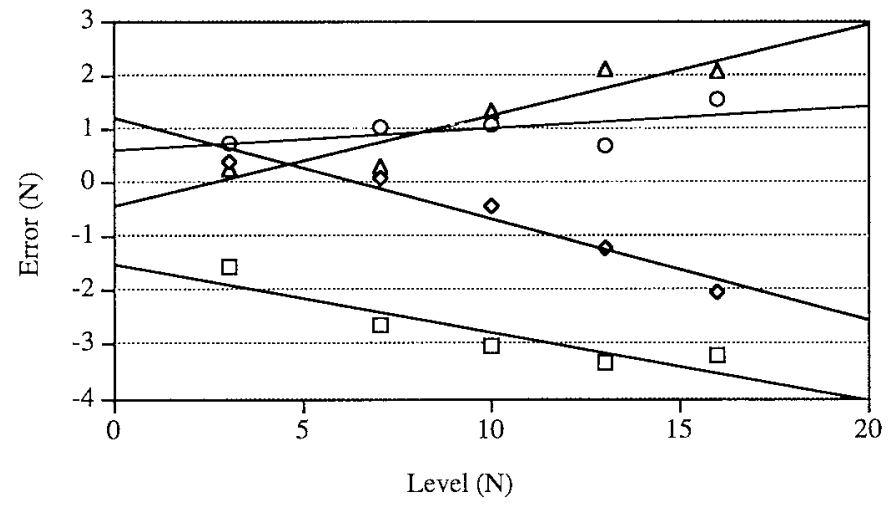

Fig. 9. Error trends vary by load and subject. Three of the four subjects display an error which increases in magnitude with increasing load. $\square$ - subject $1, \diamond$-subject 2 , ०—subject 3 , and $\triangle$-subject 4 .

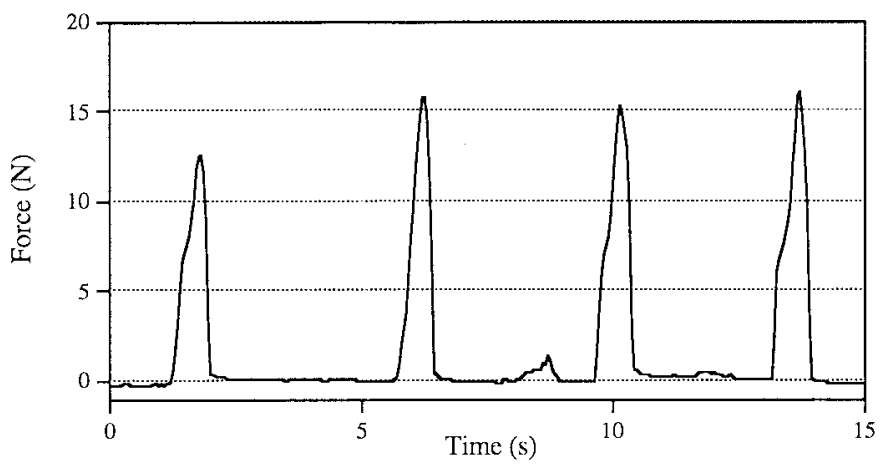

Fig. 10. The force measured by the prototype sensor while the subject placed four covers on four canisters.
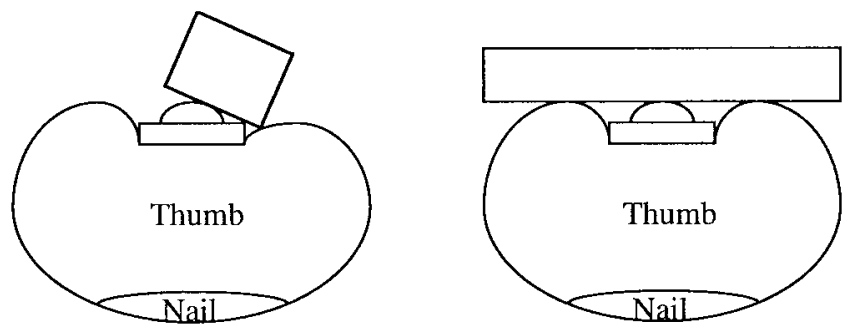

Fig. 11. Some of the applied force may bypass the sensor leading to errors in force measurement.

diameter with the force sensitive portion measuring $4 \mathrm{~mm}$ centered within the 8-mm outer dimension. For all subjects, the 8-mm diameter was significantly smaller then the width of the thumb at the mounting location (minimum thumb width $23 \mathrm{~mm}$ ). Thus, as the applied force increases the sensor begins to sink into the underlying tissue and a portion of the applied load bypasses the sensor entirely. This phenomena of force bypass can occur in different ways as illustrated in Fig. 11. With the short dome, the majority of the applied force bypasses the sensor at lower forces. Fig. 5 illustrates this phenomena. As the applied force increases, an increasing portion of the load is "seen" by the sensor and the response curve starts to approach the slope obtained with the taller dome. In all cases, the net result is an error in the force measurement since the sensor sees only a portion of the total applied load. As the 
results in Fig. 5 show, this problem is minimized by using a taller dome. A taller dome, however, can become obtrusive. A better solution is to cover the entire contact area with an array of closely spaced sensors with short domes. The fabrication techniques used to build the sensor presented in this study make this design possible and arrays of sensors are currently being designed.

\section{B. Durability}

The durability of the sensor and sensor package is critical to use in biomedical applications. A finger- or hand-mounted sensor is subjected to a wide range of forces, temperatures, and chemicals. Previous work has demonstrated package durability of approximately one day using a standard rectangular lead configuration [28]. The primary failure mechanism was lead degradation at the interface between the rigid silicon sensing element and the flexible polyimide package. Closer examination revealed that the silicon taper at the sensing element periphery [shown in Fig. 1(c)] undergoes a sequential cracking process with increasing loads. This cracking produces excessive stress in the leads immediately above the crack and eventually leads to lead failure. The use of arc lead patterns as shown in Fig. 1(a) maximizes the lead area at the sensing element periphery. Based on previous visual inspection of the cracking process, it seems unlikely that a single crack will traverse the entire arc. Thus, for lead failure to occur two or more cracks must link to completely open a lead. Also, the addition of the epoxy/urethane support material minimizes the cracking, further enhancing durability. The arc lead patterns were used in subsequent sensor fabrication, but quantitative durability tests have not been performed.

\section{Sensor Performance}

Jensen et al. [9] performed similar experiments using conductive polymer sensors. Individual finger forces were measured and compared to the total force measured by a dynamometer. For the index finger, they reported an average error of $1.0 \mathrm{~N}$, maximum error of $3.2 \mathrm{~N}$, and a standard deviation of $0.8 N$, for a $0-30-N$ total force range. The diameter of the sensing element used was $12 \mathrm{~mm}$ compared to 4-mm diameter of the silicon sensor described in this study. The errors listed in Table II are similar to those reported by Jensen et al. [9]. This is encouraging since the sensor system described here is new, and we believe improvements are possible. Conductive polymer sensors, however, have been thoroughly investigated and further improvement in performance is unlikely.

The statistical analysis of both the calibration and tracking data show significant subject-to-subject variability. There are several reasons for this variability including the experimental methodology used and the anatomical differences between subjects. As described above, the subjects were given minimal instructions during both the calibration and tracking sessions. The subjects were told only to be sure that the sensor was in contact with the dynamometer. The subjects were free to use any pinch configuration (for example, they were free to use one or more opposing fingers in contact with the dynamometer). The freedom of the subject to pinch the dynamometer in a variety of ways may have contributed to the subject variability and to the variability within a subject. The subjects were given a chance to practice pinching the dynamometer before any data was collected. It was observed that subjects tended to choose a pinch configuration and then stick with it throughout the experiment. This would indicate that the lack of specific pinch instructions contributed mainly to the variability between subjects.

The mechanical characteristics of the finger varies between subjects. For example, the characteristics of a finger pad for a construction worker are, typically, significantly different than for an office worker. A construction worker may have calloused skin which provides a much stiffer backing for the sensor then the backing provided by the subtle finger pad of an office worker. These differences may give rise to variability in sensor performance between subjects and between bench (Fig. 2) and human subject [Fig. 6(a)] experiments due to different dynamics of the force bypass phenomena discussed above.

The positive error present in subject 2 during the tracking experiment may indicate that the subject used a different pinch configuration for the calibration runs than during the tracking runs. This is interesting because the experiments were both performed during one experimental session lasting 15-30 min. The only difference between the two experiments from a procedural perspective was the rate of loading. That is, for the dynamic calibration experiment the subjects pinched the dynamometer with a fairly constant loading and unloading rate, while for the tracking experiment the subjects were required to hold a specified level for a few seconds. In addition, they were able to freely vary the rate of loading when moving between levels. It is possible that theses differences lead to significantly different pinch configurations. For example, the subjects who exhibited positive errors during the tracking experiment may have been using a pinch configuration that exhibited high bypass errors during the dynamic calibration experiment. When they changed their pinch configuration for the tracking experiments less force bypassed the sensor leading to positive errors. A similar argument can be made for the subjects showing a negative error.

The error trends displayed in Fig. 9 indicate an influence of load magnitude on error and the error trends are also subject dependent. Two subjects showed an increasing negative error, one subject showed an increasing positive error, and one subject showed no obvious error trend. These trends are further evidence of differing pinch configurations and anatomical differences between subjects.

An array of closely spaced sensors has the potential to overcome these problems. For loads that are large (in terms of contact area) relative to the sensor spacing, the use of an array of sensors will minimize the force bypass phenomena and, in addition, reduce the effect of differences in mechanical characteristics between subjects on sensor output. The use of arrays which cover the entire contacting area would also provide information about load shapes and pinch/grasp configurations. Finally, an array which covers the entire finger pad will ensure that all of the applied load is sensed and provide pressure (force distribution) information. 


\section{Temperature Effects}

Silicon piezoresistors exhibit significant drift with temperature. Although a Wheatstone bridge configuration helps to minimize temperature effects, compensation is still necessary in many applications. Since the experiments described here were performed in a controlled laboratory setting, temperature compensation was not necessary. For use in FNS systems, where the sensor may be exposed to widely varying temperatures, temperature compensation may be necessary. Many methods of temperature compensation are available [36], [37]. Ideally temperature compensation should be included on the sensor itself. The necessary temperature compensation circuits can be fabricated on the sensor away from the diaphragm, thus, the compensation circuit is subjected to the same temperature changes as the bridge.

\section{CONCLUSION}

While many descriptions of silicon-based tactile sensors exist in the literature, few were designed for use on the human skin. Sorab et al. [10] modified a commercially available silicon pressure sensor for use in the hand and Neuman et al. [27] and Ko et al. [26] developed silicon-based force sensors for biomedical applications. In both cases, the resulting sensors were too bulky for long-term use on the hand. The sensor described utilizes a polyimide-based packaging scheme to incorporate a silicon-based sensor in flexible skin which facilitates use at the skin/load interface. The results of testing on human subjects is encouraging. Current and future work include finite element modeling of sensing elements capable of shear force measurement and the fabrication of arrays of closely spaced sensing elements in the polyimide skin.

\section{ACKNOWLEDGMENT}

The authors would like to thank D. Christensen, for many helpful discussions. They would also like to thank K. Cohen and E. Welch for help with data acquisition and analysis. The authors are grateful to DuPont Co., Wilmington, DE, for providing all of the polyimides used in this work.

\section{REFERENCES}

[1] P. Dario, "Tactile sensing: Technology and applications," Sens. Actuators A, 25-27, pp. 251-256, 1991 .

[2] P. P. L. Regtien, “Tactile imaging," Sens. Actuators A, 31, pp. 83-89, 1992.

[3] J. G. Webster, Ed., Tactile Sensors for Robotics and Medicine. New York: Wiley, 1988.

[4] P. E. Crago, H. J. Chizeck, M. R. Neuman, and T. F. Hambrecht, "Sensors for use with functional neuromuscular stimulation," IEEE Trans. Biomed. Eng., vol. BME-33, pp. 256-267, 1986.

[5] K. N. An, E. Y. Chao, W. P. Cooney, and R. L. Linscheid, "Forces in the normal and abnormal hand," J. Orthopaed. Res., vol. 3, pp. 202-211, 1985.

[6] R. A. Dickson, A. Petrie, F. V. Nicolle, and J. S. Calnan, "A device for measuring the forces of the digits of the hand," Biomed. Eng., vol. 7, pp. 270-273, 1972.

[7] J. G. Webster, "Artificial sensors suitable for closed-loop control of FNS," in Neural Prostheses: Replacing Motor Function After Disease or Disability, R. B. Stein, P. H. Peckham, and D. B. Popovic, Eds. New York: Oxford Univ. Press, 1992, pp. 88-98.

[8] A. Mokshagundam, "Sensor application to peripheral neuropathy of the hand," in Tactile Sensors for Robotics and Medicine, J. G. Webster, Ed. New York: Wiley, 1988, pp. 299-307.
[9] T. R. Jensen, R. G. Radwin, and J. G. Webster, "A conductive polymer sensor for measuring external finger forces," J. Biomech., vol. 24, pp. 851-858, 1991

[10] J. Sorab, R. H. Allen, and B. Gonik, "Tactile sensory monitoring of clinician-applied forces during delivery of newborns," IEEE Trans. Biomed. Eng., vol. 35, pp. 1090-1093, 1988.

[11] R. R. Reston and J. E. S. Kolesar, "Robotic tactile sensor array fabricated from a piezoelectric polyvinylidene fluoride film," in Proc. IEEE 1990 National Aerospace and Electronics Conference (NAECON), pp. $1139-1144$

[12] F. Germagnoli, S. Lazzari, R. Lombardi, and G. Magenes, "An artificial tactile sensor for fine-form discrimination," presented at 17th Annu. Int. Conf. IEEE Eng. Med. Biol. Soc., Montreal, P.Q., Canada, 1995.

[13] D. DeRossi, A. Nannini, and C. Domenici, "Biomimetic tactile sensor with stress-component discrimination capability," J. Molecular Electron., vol. 3, pp. 173-181, 1987.

[14] G. L. Fellows and A. Freivalds, "The use of force sensing resistors in ergonomic tool design," in Proc. Human Factors Society 33rd Annu. Meeting, 1989, pp. 713-717.

[15] R. A. Pax, J. G. Webster, and R. G. Radwin, A Pressure Sensing Glove Using Conductive Polymer Pressure Sensors. Madison, WI: Univ. Wisconsin Press, 1989.

[16] K. A. Kaczmarek, K. M. Kramer, J. G. Webster, and R. G. Radwin, “A 16-channel 8-parameter waveform electrotactile stimulation experiment system," IEEE Trans. Biomed. Eng., vol. 38, pp. 933-943, 1991.

[17] R. G. Radwin and S. Oh, "External finger forces in submaximal fivefinger static pinch prehension," Ergonom., vol. 35, pp. 275-288, 1992.

[18] L. I. Automation, Lord Series 210 Tactile Sensing System, Covy, NC: 1987.

[19] M. R. Neuman, P. E. Crago, P. H. Peckham, R. Riso, H. J. Chizek, and C. V. Doren, Artificial Sensory Transducers, National Institute of Neurological Disorders and Stroke, 1991.

[20] K. Suzuki, K. Najafi, and K. D. Wise, "A 1024-element highperformance silicon tactile imager," IEEE Trans. Electron Devices, vol. 37, pp. 1852-1859, 1990

[21] Samaun, K. D. Wise, and J. B. Angell, "An IC piezoresistive pressure sensor for biomedical instrumentation," IEEE Trans. Biomed. Eng., vol. BME-20, pp. 101-109, 1973.

[22] Y. S. Lee and K. D. Wise, "A batch-fabricated silicon capacitive pressure transducer with low temperature sensitivity," IEEE Trans. Electron Devices, vol. ED-29, pp. 42-48, 1982.

[23] H. Tanigawa, T. Ishihara, M. Hirata, and K. Suzuki, "MOS integrated silicon pressure sensor," IEEE Trans. Electron Devices, vol. ED-32, pp. 1191-1195, 1985.

[24] B. Kane and G. Kovacs, "A CMOS compatible traction stress sensing element for use in high resolution tactile imaging," in Proc. 8th Int. Conf. Solid-State Sensors and Actuators, and Durosensors IX, Stockholm, Sweden, 1995, pp. 648-651.

[25] Z. Chu, P. M. Sarro, and S. Middelhoek, "Silicon three-axial tactile sensor," in Proc. 8th Int. Conf. Solid-State Sensors and Actuators and Eurosensors IX, Stockholm, Sweden, 1995, pp. 656-659.

[26] W. H. Ko, J. Hynecek, and S. F. Boettcher, "Developement of a miniature pressure transducer for biomedical applications," IEEE Trans. Electron Devices, vol. ED-26, pp. 1896-1905, 1979.

[27] M. R. Neuman and J. R. Buckett, "Thumb force and position sensors," in Proc. 35th Annu. Conf. Eng. Med. Biol., Philadelphia, PA, 1982, p. 122.

[28] D. J. Beebe and D. D. Denton, "A flexible polyimide-based package for silicon sensors," Sens. Actuators A, 44, pp. 57-64, 1994.

[29] D. J. Beebe, A. S. Hsieh, D. D. Denton, and R. G. Radwin, "A silicon force sensor for robotics and medicine," Sens. Actuators A, 50, pp. 55-65, 1995.

[30] P. W. Barth, S. L. Bernard, and J. B. Angell, "Flexible circuit and sensor arrays fabricated by monolithic silicon technology," IEEE Trans. Electron Devices, vol. ED-32, pp. 1202-1205, 1985.

[31] P. A. Payne, "Measurement of properties and function of skin," Clin Phys. Physiol. Meas., vol. 12, pp. 105-129, 1991.

[32] B. Finlay, "Dynamic mechanical testing of human skin in vivo," J. Biomech., vol. 3, pp. 557-568, 1970.

[33] J. M. Pereira, "Dynamic measurement of the viscoelastic properties of skin," J. Biomech., vol. 24, pp. 157-162, 1991.

[34] R. G. Radwin, G. P. Masters, and F. W. Lupton, "A linear forcesumming hand dynamometer independent of point of application," Appl. Ergonom., vol. 22, pp. 339-343, 1991.

[35] G. Box, W. Hunter, and J. Hunter, Statistics for Experimenters. New York: Wiley, 1978.

[36] S. Middelhoek and S. A. Audet, Silicon Sensors. London, U.K. Academic, 1989

[37] D. Tandeske, Pressure sensors: Selection and Application. New York: Marcel Dekker, 1991. 


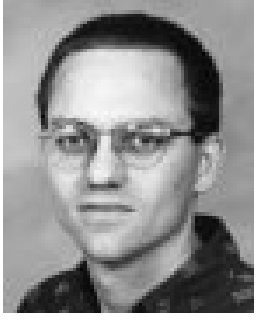

David J. Beebe (S'89-M'90) received the B.S., M.S., and Ph.D. degrees in electrical engineering in 1987, 1990, and 1994, respectively, from the University of Wisconsin-Madison.

He was an electrical engineer for Kimberly-Clark Corp. from 1987 to 1989 . He is an Assistant Professor in the Department of Electrical and Computer Engineering and an Assistant Research Professor in the Beckman Institute at the University of Illinois at Urbana-Champaign. He From 1991 to 1994, he was an NIH Biotechnology Predoctoral Trainee. From 1994 to 1996, he was an Assistant Professor at Louisiana Technical University, Ruston. During the summer of 1992, he worked at Medtronic, Inc., Minneapolis, MN, and developed new hemodynamic sensing concepts. His broad interests are in biomedical instrumentation and the development of microfabricated devices for biomedical applications. His current interest include technology development for the labeling and handling of biological objects, development of devices for the separation and detection of biological macromolecules, development of electrostatic haptic displays, and continued work on tactile sensors.

Dr. Beebe is a contributing author to Biomedical Digital Signal Processing, W. J. Tompkins, Ed. (Englewood Cliffs, NJ: Prentice-Hall, 1993), to Prevention of Pressure Sores, J. G. Webster, Ed. (Bristol, England: Adam Hilger, 1991), and to Occupational Ergonomics, A. Bhattacharya and J. D. McGlothlin, Eds. (New York: Marcel Dekker, 1996).

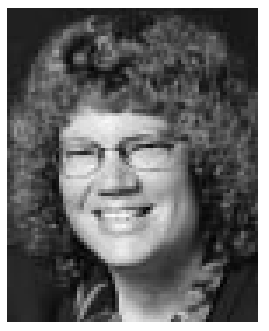

Denice D. Denton (S'83-M'85) received the B.S and M.S. degrees in 1982, and the Ph.D. degree in 1987 in electrical engineering from Massachusetts Institute of Technology (MIT), Cambridge. Her dissertation focused on the characterization of polyimide, a polymeric insulating material used in integrated circuits as an intermetal insulator and passivant. Particular emphasis was placed on monitoring the effects of moisture on the dielectric properties of this film. In the course of this work, she began to investigate the possibility of the use of polyimide as a capacitive moisture sensor.

She is the Dean of Engineering and a Professor in the Department of Electrical Engineering at the University of Washington, Seattle. She was a Professor of Electrical and Computer Engineering at the University of Wisconsin (UW)-Madison, from 1987 to 1996. She spent the fall semester of 1991 as a Visiting Scientist and the summer of 1993 as a Visiting Professor at the Swiss Federal Institute of Technology (ETH), Zürich, Switzerland. Her current interests include plasma deposition of polymers used in photonics applications, the investigation of the long-term reliability implications of the use of polymers in integrated circuit applications, and the use of micromachining in solid-state actuator design. She headed the Plasma Deposition and Polymerization Thrust Area of the NSF Engineering Research Center (ERC) for Plasma-Aided Manufacturing at the University of Wisconsin-Madison from 1991 to 1996.

Professor Denton was Co-Director of the National Institute for Science Education in 1995-1996. She is a recipient of the National Science Foundation Presidential Young Investigator Award (1987-1992) and Digital Equipment Corporation Faculty Grant (1990-1991). She has also won the University of Wisconsin Kiekhofer Distinguished Teaching Award (1990), and the Benjamin Smith Reynolds Teaching Award, UW College of Engineering (1994), and is a member of the Teaching Academy, UW-Madison (1994). In addition, she is the recipient of the American Society of Engineering Education AT\&T Foundation Teaching Award (1991), the Eta Kappa Nu, C. Holmes MacDonald Distinguished Young Electrical Engineering Teaching Award (1993), the W. M. Keck Foundation Engineering Teaching Excellence Award (1994), the ASEE George Westinghouse Award (1995), and the IEEE Harriet B. Rigas Teaching Award (1995). She is a member of the National Academy of Sciences/National Research Council Board on Engineering Education (1991-1996) and the NAS/NRC Committee on Undergraduate Science Education (1993-1997). She has developed a Microfabrication Demonstration Kit which is being used in K-12 classrooms in more than 30 states to introduce students to microelectronics. She also works actively to encourage women and under-represented minorities to consider careers in science and engineering.

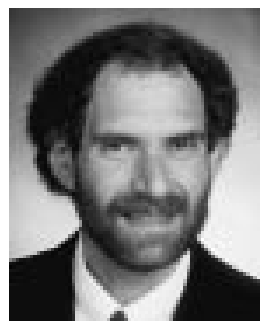

Robert G. Radwin (S'84-M'85-SM'94) received the B.S. degree in electrical engineering from the Polytechnic Institute of New York, Brooklyn in 1975, and M.S. degrees in electrical and computer engineering and in bioengineering from the University of Michigan, Ann Arbor, in 1979. He also received the $\mathrm{Ph} . \mathrm{D}$. degree from the University of Michigan in 1986, where he was a Postdoctoral Research Fellow at the Center for Ergonomics (1987).

$\mathrm{He}$ is currently a Professor at the University of Wisconsin-Madison, where he conducts research and teaches in the areas of ergonomics, human factors, and biomedical engineering, and directs the interdisciplanary program in biomedical engineering. He actively studies the recognition, causes, and control of cumulative trauma disorders in manual work. His research is concerned with developing analytical methods for measuring and assessing exposure to physical stress in the work place, ergonomics guidelines for the design, selection, installation and use of manually operated equipment, and functional deficits associated with musculoskeletal disorders and peripheral neuropathies.

Dr. Radwin is the recipient of a Presidential Young Investigator Award from the National Science Foundation and a Special Emphasis Research Career Award from the National Institute for Occupational Safety and Health. He is a member of Alpha Pi Mu, Eta Kappa Mu, and Sigma Xi.

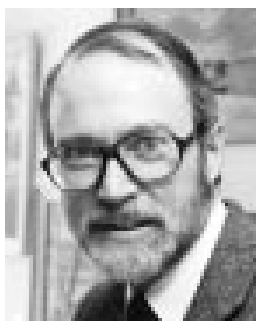

John G. Webster (M'61-SM'71-F'86-LF'97) received the B.E.E. degree from Cornell University, Ithaca, NY, in 1953, and the M.S.E.E. and Ph.D. degrees from the University of Rochester, Rochester, NY, in 1965 and 1967, respectively.

He is Professor of Electrical and Computer Engineering at the University of Wisconsin-Madison. He teaches undergraduate and graduate courses in the field of medical instrumentation and does research on RF cardiac ablation and measurement of vigilance. He is author of Transducers and Sensors, an IEEE/EAB Individual Learning Program (Piscataway, NJ: IEEE, 1989). He is coauthor, with B. Jacobson, of Medicine and Clinical Engineering (Englewood Cliffs, NJ: Prentice-Hall, 1977), and with R. Pallás-Areny, of Sensors and Signal Conditioning (New York: Wiley, 1991). He is Editor of Encyclopedia of Medical Devices and Instrumentation (New York: Wiley, 1988), Tactile Sensors for Robotics and Medicine (New York: Wiley, 1988), Electrical Impedance Tomography (Bristol, England: Adam Hilger, 1990), Teaching Design in Electrical Engineering (Piscataway, NJ: Educational Activities Board, IEEE, 1990), Prevention of Pressure Sores: Engineering and Clinical Aspects (Bristol, England: Adam Hilger, 1991), Medical Instrumentation: Application and Design, 3rd ed. (New York: Wiley, 1997), and Design of Cardiac Pacemakers (Piscataway, NJ: IEEE Press, 1995). He is CoEditor, with A. M. Cook, of Clinical Engineering: Principles and Practices (Englewood Cliffs, NJ: Prentice-Hall, 1979) and Therapeutic Medical Devices: Application and Design (Englewood Cliffs, NJ: Prentice-Hall, 1982), with W. J. Tompkins, of Design of Microcomputer-Based Medical Instrumentation (Englewood Cliffs, NJ: Prentice-Hall, 1981) and Interfacing Sensors to the IBM PC (Englewood Cliffs, NJ: Prentice-Hall, 1988), and with A. M. Cook, W. J. Tompkins, and G. C. Vanderheiden, of Electronic Devices for Rehabilitation (London: Chapman \& Hall, 1985).

Dr. Webster has been a member of the IEEE-EMBS Administrative Committee and the NIH Surgery and Bioengineering Study Section. He is a Fellow of the Instrument Society of America and the American Institute of Medical and Biological Engineering. He is the recipient of the AAMI Foundation Laufman-Greatbatch Prize and the ASEE/Biomedical Engineering Division, Theo C. Pilkington Outstanding Educator Award. 